characteristics associated with the efficacy of face to face interventions.

Methods Cochrane Controlled Register of Trials, Embase, MEDLINE (ovid), PsycINFO, Web of science, PubMed, and Scopus databases were searched (from start date till May 2019). Randomized Controlled Trials (RCTs) were included if they described the intervention to improve medication adherence delivered via face to face; included any LTHC, included a comparator group, conducted in any setting and published in English language. Studies were excluded if used additional delivery mode (e.g. leaflet, SMS, apps, follow up phone call related to medication adherence), involved adolescents $(<18$ years), children, peers, family members and used group format. Two reviewers independently assessed studies for inclusion, appraised risk of bias and extracted data. Pooled effect sizes will be calculated using random/fixed effects model using RevMan 5.3 software.

Results Results from 50 studies were included in the analysis $(n=10576)$. Most face to face interventions took place in secondary care $(n=26)$, included pharmacists in delivery $(n=12)$ and involved counselling $(n=10)$ and behavioural $(n=8)$ approaches on multiple occasions. Majority of the studies were published in years 2014-2019 $(n=26)$ and conducted in the USA $(n=16)$. Most common health condition was HIV $(n=10)$ in comparison to other LTHCs. The first follow up time point (related to medication adherence outcome), will be analysed from all included studies. In terms of risk of bias, most studies were rated as having overall high risk of bias $(n=37)$, followed by some concern due to lack of information $(n=12)$ and low risk of bias $(n=1)$. BCTs were only used in the intervention groups $(n=18)$, in which most commonly used were: 'self-monitoring behaviour' and 'action planning'. The impact of specific individual BCTs and BCTs domains on effectiveness will be examined. Subgroup analyses will be conducted related to age and gender. Results related to the aims of this meta-analysis and meta-regression will be available by the time of the conference.

Conclusion Efficacy of these interventions related to medication adherence outcome and core components of face to face consultations with BCT coding could be very useful to design a cost and time effective face to face very brief or brief interventions related to medication adherence to be implemented in primary care practices in the future.

\section{P41 ARTERIAL STIFFNESS PROGRESSION AND RISK OF MAJOR ADVERSE CARDIOVASCULAR EVENTS ACCORDING TO HYPERTENSION STATUS IN A COHORT OF BRITISH CIVIL SERVANTS}

\begin{abstract}
${ }^{1} \mathrm{CA}$ Valencia-Hernández* ${ }^{2} \mathrm{C}$ McEniery, ${ }^{2} \mathrm{IB}$ Wilkinson, ${ }^{3} \mathrm{~S}$ Ahmadi-Abhari, ${ }^{4} \mathrm{~J}$ Lindbohm, ${ }^{1}$ MJ Shipley, ${ }^{1}$ EJ Brunner. ${ }^{1}$ Research Department of Epidemiology and Public Health, University College London, London, UK; ${ }^{2}$ Division of Experimental Medicine and Immunotherapeutics, University of Cambridge, Cambridge, UK; ${ }^{3}$ Ageing Epidemiology Research Unit, Imperial College London, London, UK; ${ }^{4}$ Clinicum, Department of Public Health, University of Helsinki, Helsinki, Finland
\end{abstract}

\subsection{6/jech-2020-SSMabstracts. 135}

Background Arterial stiffness is defined as the loss of compliance of the elastic arteries in the human body and is associated to ageing. Hypertension is the leading global risk factor for cardiovascular disease and is related to a higher frequency of cardiovascular events. A high proportion of the hypertensive population is not aware of their condition or is not provided with adequate blood pressure treatment. The aim of this work is to assess the 4-year rate of change in arterial stiffness according to antihypertensive treatment at baseline in a population of British civil servants and to estimate the differences in the risk of major cardiovascular events between the different categories of change.

Methods Carotid-femoral Pulse Wave Velocity (cf-PWV) is the gold-standard to assess arterial stiffness and it was measured both at baseline (Phase 9, 2008-9) and follow-up (Phase 11, 2011-12) in 4998 participants of the cohort (3680 men; 1318 women). It was measured using the Sphygmacor ${ }^{\circledR}$ Atcor tonometric device. Major cardiovascular events were defined as myocardial infarction, stroke and coronary heart disease. The information about these outcomes was extracted from the NHS Hospital Episode Statistics. 5-year change models were fitted using linear mixed model regression.

Results There were 1842 (36.9\%) controlled hypertensive, 871 (17.4\%) untreated hypertensive and 557 (11.1\%) uncontrolled hypertensive participants in the total sample. A model adjusted for sociodemographic characteristics, comorbidities and health behaviours showed that compared to non-hypertensives, mean PWV increase was $0.04 \mathrm{~m} / \mathrm{s}$ (95\%CI: $-0.04,0.17 \mathrm{p}: 0.51)$ for controlled hypertensives, $\quad 0.20 \mathrm{~m} / \mathrm{s} \quad$ (95\%CI: $0.06,0.35$ $\mathrm{p}<0.001)$ untreated hypertensives and 0.25 (95\%CI: $0.03,0.47$ $\mathrm{p}<0.05)$ for uncontrolled hypertensives. The risk of major adverse cardiovascular events was almost four times in uncontrolled hypertensive participants (HR: 3.72; 95\%CI 2.47-5.59) and three times in controlled hypertensives (HR: 2.48 ; 95\%CI 1.92-3.21) compared to normotensive participants. A significant difference was not found in untreated hypertensive participants.

Conclusion The rate of arterial stiffening over time and the risk of major adverse cardiovascular effects is higher in uncontrolled participants of the Whitehall II study, compared with normotensive participants. This is additional evidence of the need for improved strategies for blood pressure control in hypertensive patients.

\section{P42 MATERNAL CHRONIC HYPERTENSION AND THE RISK OF ADVERSE MATERNAL AND BIRTH OUTCOMES: A POPULATION-BASED STUDY}

${ }^{1,2} \mathrm{EJ}$ O'Reilly, ${ }^{3,4} \mathrm{FP} \mathrm{McCarthy},{ }^{5} \mathrm{M}$ Kublickas, ${ }^{6} \mathrm{~K}$ Kublickiene, ${ }^{1,3} \mathrm{AS}$ Khashan, ${ }^{1,3} \mathrm{SY}$ Al Khalaf* ${ }^{*}$ 'School of Public Health, University College Cork, Cork, Ireland; ${ }^{2}$ Department of Nutrition, Harvard T.H. Chan School of Public Health, Boston, MA, USA; ${ }^{3}$ INFANT Research Centre, University College Cork, Ireland; ${ }^{4}$ Department of Obstetrics and Gynaecology, University College Cork, Cork, Ireland; ${ }^{5}$ Department of Obstetrics and Gynaecology, Karolinska Institutet and Karolinska University hospital Stockholm, Sweden; ${ }^{6}$ Renal Medicine, Department of Clinical intervention, Science and Technology (CLINTEC), Karolinska Institutet and Karolinska University hospital, Stockholm, Sweden

\subsection{6/jech-2020-SSMabstracts. 136}

Background Chronic hypertension $(\mathrm{CH})$ has been linked with adverse pregnancy outcomes, but it is unclear whether these associations are changing by maternal characteristics or over time.

The objective of this study was to examine the association between maternal $\mathrm{CH}$ and adverse pregnancy outcomes, and to determine whether the risk varies over time. We also aimed to assess the associations according to maternal age (younger or $\geq 35$ yrs.) and other maternal characteristics.

Methods This population-based cohort study included women who had singleton births in Sweden between 1982 and 2012. 